\title{
Measurements of nuclear magnetic moments and electric quadrupole moments of Lu isotopes
}

\author{
C. König, B. Hinfurtner, E. Hagn, and E. Zech \\ Physik-Department, Technische Universität München, D-85748 Garching, Germany \\ R. Eder \\ PPE Division, CERN, CH-1211 Geneva 23, Switzerland \\ (ISOLDE Collaboration) \\ (Received 18 April 1996)
}

\begin{abstract}
Nuclear magnetic resonance measurements were performed on the ground states of ${ }^{169} \mathrm{Lu},{ }^{171} \mathrm{Lu},{ }^{172} \mathrm{Lu}$, ${ }^{173} \mathrm{Lu}$, and ${ }^{177} \mathrm{Lu}$ and on the $23 / 2^{-}$isomeric state ${ }^{177 m} \mathrm{Lu}$. The radioactive isotopes were implanted into hcp Co single crystals using the mass separator ISOLDE at CERN. For ${ }^{169} \mathrm{Lu},{ }^{171} \mathrm{Lu},{ }^{172} \mathrm{Lu},{ }^{173} \mathrm{Lu}$, and ${ }^{177} \mathrm{Lu}$ the quadrupole subresonance structure could be resolved well. With the magnetic hyperfine fields and the electric field gradient of $\mathrm{LuCo}$ (hcp) derived from the results on ${ }^{177} \mathrm{Lu}$, the magnetic moments and electric quadrupole moments of ${ }^{169} \mathrm{Lu},{ }^{171} \mathrm{Lu},{ }^{172} \mathrm{Lu}$, and ${ }^{173} \mathrm{Lu}$ were determined. The nuclear moments of the high-spin isomer ${ }^{177 m} \mathrm{Lu}$ were determined with a resonance-offset technique. [S0556-2813(96)02109-7]

PACS number(s): 21.10.Ky, 27.70.+q, 76.60.Jx, 76.80.+y
\end{abstract}

\section{INTRODUCTION}

The nuclear magnetic moments of neutron-deficient $\mathrm{Lu}$ isotopes have been the subject of several investigations and speculations [1-4]. Krane et al. reported measurements of the magnetic moments of ${ }^{171,172} \mathrm{Lu}[1],{ }^{173,174 g, 174 m} \mathrm{Lu}$ [2], and ${ }^{177 m} \mathrm{Lu}[3]$. As the region $A \sim 170$ is characterized by a stable prolate deformation with $\beta \approx 0.25$, it is expected that the magnetic moments of the single-particle ground states and the low-lying three-particle states of the odd isotopes and the two-particle ground states and low-lying isomeric states of the even isotopes are well described by the Nilsson model. This expectation seemed to be fulfilled on the whole, albeit with discrepancies observed between experimental and theoretical magnetic moments. Especially for the $6^{-}$highspin isomer ${ }^{174 m} \mathrm{Lu}$ the experimental magnetic moment differed conspicuously from the value which was predicted from magnetic properties of the rotational band built up on the $6^{-}$level $[5,6]$. This led to the speculation that the additivity of magnetic moments could be violated in ${ }^{174 m} \mathrm{Lu}$ [5]. A minor discrepancy was also apparent for the three-particle $23 / 2^{-}$state ${ }^{177 m} \mathrm{Lu}[3,4]$.

As the actual existence of an additivity violation of the magnetic moments would have severe consequences for the understanding of the magnetism in nuclei in general, Drissi et al. [7] revisited the problem of ${ }^{174 m} \mathrm{Lu}$. They finally concluded that nuclear orientation (NO) might be an improper technique for the determination of magnetic moments of $\mathrm{Lu}$ isotopes.

In the "standard" low-temperature nuclear orientation technique the radioactive isotope of interest is embedded into a ferromagnetic environment in which a large magnetic hyperfine field acts on the embedded "impurities." At low temperatures the system becomes oriented which is accompanied by an anisotropic angular distribution of the emitted $\gamma$ rays. The $\gamma$ anisotropy depends on the hyperfine splitting frequency $\nu_{M}$, which is the product of the $g$ factor and the hyperfine field, and on the temperature $T$. The $\gamma$ anisotropy is measured as a function of $T$, from which $\nu_{M}$ can be de- termined, normally with a least-squares fit. This method is an integral technique, which means that, for the determination of the $g$ factor or the magnetic moment, the distribution of hyperfine fields acting on the impurity nuclei has to be known. If all impurity nuclei are substituted onto regular lattice sites, the interpretation of the measured $\gamma$ anisotropies is straightforward and unique. In reality, the impurity nuclei are often substituted onto the regular sites not with $100 \%$ but with a large fraction $f$. Then a "two-site model" may be applied for the interpretation. Within this model it is assumed that the fraction of nuclei $f$ is subject to the full hyperfine field, while the residual fraction of nuclei, $1-f$, is subject to a negligibly small hyperfine interaction. This is a moderately good approximation in many cases.

In alloying or implanting Lu as dilute impurity in "standard" ferromagnetic hosts, such as Fe and Co, the Lu atoms are substituted onto regular lattice sites only with a small fraction. The residual $\mathrm{Lu}$ atoms are substituted onto lattice sites, at which the average hyperfine field is smaller than the substitutional hyperfine field, but significantly different from zero. The exact location of $\mathrm{Lu}$ at these low-field sites is unknown. This complicates the interpretation of $\gamma$ anisotropies considerably. Therefore, Krane et al. [1-3] used $\mathrm{ZrFe}_{2}$ as a matrix, which had been found to be a good matrix for the neighboring $\mathrm{Hf}$, which is chemically homologous to $\mathrm{Zr}$ (but not to $\mathrm{Lu}$ ). They found, however, that Lu has a similarly bad substitution behavior in $\mathrm{ZrFe}_{2}$ as in Fe. This means that the distribution of the hyperfine field is unkown. Krane et al. took into account this uncertainty by simultaneous measurements of different isotopes and extracting only ratios of magnetic moments. This is a good procedure if nuclear states with the same spin and not too different magnetic moments are involved. If, however, the magnetic moments and the decay properties of the two isotopes are very different, such a procedure fails, as the weighting of the different field components is then different.

Thus, among the different low-temperature nuclearorientation methods, only a differential technique, such as NMR-ON (nuclear magnetic resonance on oriented nuclei) is 
expected to yield unambiguous results for the nuclear magnetic moments.

The first successful NMR-ON experiment on ${ }^{177} \mathrm{Lu}$ in $\mathrm{Fe}$ was reported by Herzog et al. [8]. In this experiment the resonance width $\Gamma$ was very broad $[\Gamma / \nu=0.058(4)]$. Recently, the NMR-ON resonance of ${ }^{174 m} \mathrm{LuFe}$ was detected [9]; actually a magnetic moment was obtained which is completely different from the NO result, removing the discrepancies concerning additivity violation. In the ${ }^{174 m} \mathrm{LuFe}$ NMR-ON experiment the resonance was also relatively broad, $\Gamma / \nu=0.053(9)$, indicating that the broad distribution of the hyperfine field at substitutional sites is an inherent property of the LuFe system. For precision measurements of magnetic moments a system with a smaller field distribution would be desirable. Therefore, in the present work, hcp Co was used as a host matrix for Lu for the first time. We found that the relative linewidths are smaller, and that, in addition, the electric field gradient of $\mathrm{Lu}$ in hcp Co is so large that the quadrupole splittings could be resolved up to $I=4$. In this way, the magnetic moments and electric quadrupole moments for radioactive $\mathrm{Lu}$ isotopes could be determined simultaneously. For the $23 / 2^{-}$high-spin isomer ${ }^{177 m} \mathrm{Lu}$ the quadrupole interaction could not be resolved; in this case, the quadrupole splitting was determined with a resonance-offset technique.

Simultaneously with our work the nuclear moments of radioactive Lu isotopes between $A=167$ and 177 were measured via laser spectroscopy (LS) by Georg et al. [10].

\section{QUADRUPOLE INTERACTION WITH hcp Co}

\section{A. Hyperfine interaction aspects of hep Co}

The hyperfine interaction of radioactive isotopes at substitutional lattice sites in ferromagnetic hcp Co consists of a magnetic-dipole and an electric-quadrupole contribution: (i) The magnetic hyperfine field $B_{\mathrm{HF}}$ is parallel (antiparallel) to the electronic magnetization $\vec{M}$, the absolute magnitude depending (slightly) on the angle between $\vec{M}$ and the singlecrystal $c$ axis. (ii) Because of the hexagonal symmetry of hcp $\mathrm{Co}$, an axially symmetric lattice electric field gradient (EFG) $e q^{(L)}$ exists, the principal axis of the EFG being given by the single-crystal $c$ axis. Depending on the type of the impurity, an additional EFG $e q^{(c)}$ may exist which is collinear with the magnetic hyperfine field. The principal axis system for this type of EFG is given by the direction of $B_{\mathrm{HF}}$. Such a "collinear' EFG may be the result of an unquenched orbital momentum, which, because of the spin-orbit interaction, points into the direction of $B_{\mathrm{HF}}$.

In the absence of an external magnetic field $B_{\text {ext }}, \vec{M}$ and $\vec{B}_{\mathrm{HF}}$ are parallel to the $c$ axis, which is the (only one) direction of spontaneous magnetization. By applying an external magnetic field $B_{\text {ext }}$ nonparallel to the $c$ axis, the direction of the magnetization can be turned away from the $c$ axis towards the direction of the external magnetic field. The direction of the magnetization with respect to $B_{\text {ext }}$ and the $c$ axis is then fixed by the minimum condition for the free energy which is the sum of magnetization energy and the crystal anisotropy energy. If $B_{\text {ext }}$ is applied perpendicular to the $c$ axis, the direction of the magnetization and hence the direction of the hyperfine field can be forced parallel to $B_{\text {ext }}$, i.e., perpendicular to the $c$ axis, if $B_{\text {ext }}$ exceeds the perpendicular anisotropy field $B_{\mathrm{A}}^{\perp}=13.4 \mathrm{kG}$. The angular dependence of the hyperfine interaction has been discussed in a recent paper [11]. Here we want to confine ourselves to the two limiting cases relevant for the measurements presented in this paper.

(i) $B_{\text {ext }} \| c$, i.e., $\theta=0^{\circ}$, where $\theta$ is the angle between the electronic magnetization $\vec{M}$ and the single-crystal $c$ axis, which we denote as " 0 ' geometry."

(ii) $B_{\text {ext }} \perp c$. In this case, $\theta=90^{\circ}$ (" $90^{\circ}$ geometry") is achieved for $B_{\text {ext }} \gtrsim 13.4 \mathrm{kG}$.

The energy eigenvalues of the nuclear spin system are calculated by diagonalization of the Hamiltonian

$$
\begin{gathered}
\mathcal{H}=\mathcal{H}_{M}+\mathcal{H}_{Q}^{(\mathrm{c})}+\mathcal{D}(\theta) \mathcal{H}_{Q}^{(\mathrm{L})} \mathcal{D}^{-1}(\theta), \\
\mathcal{H}_{M}=-h \nu_{\mathrm{mag}} I_{z} \\
\mathcal{H}_{Q}^{(c, L)}=h \nu_{Q}^{(c, L)} \frac{3 I_{z}^{2}-I(I+1)}{4 I(2 I-1)}
\end{gathered}
$$

where $\mathcal{D}(\theta)$ is the normally used rotation matrix and $\nu_{\text {mag }}$ and $\nu_{Q}^{(c, L)}$ are the magnetic and electric interaction frequencies,

$$
\begin{gathered}
\nu_{\text {mag }}=\left|g \mu_{N} B^{(\text {eff })} / h\right|, \quad \nu_{Q}^{(c)}=e^{2} q^{(c)} Q / h, \\
\nu_{Q}^{(L)}=e^{2} q^{(L)} Q / h .
\end{gathered}
$$

Here $g$ and $e Q$ are the nuclear $g$ factor and the spectroscopic quadrupole moment, and $e q^{(\mathrm{c})}$ and $e q^{(L)}$ are the "collinear" electric field gradient (axialsymmetric with respect to the direction of $B_{\mathrm{HF}}$ ) and the lattice field gradient (axialsymmetric with respect to the $c$ axis), respectively.

The effective magnetic field $B^{(\mathrm{eff})}$ is given by

$$
B^{(\text {eff })}=B_{\mathrm{HF}}+\operatorname{sgn}\left(B_{\mathrm{HF}}\right)(1+K) B_{0},
$$

where for $B_{\mathrm{HF}}$ the "parallel" hyperfine field $B_{\mathrm{HF}}^{\|}$or the " "perpendicular' hyperfine field $B_{\mathrm{HF}}^{\perp}$ has to be taken, $K$ is a parameter including Knight shift and diamagnetic shielding, $B_{0}$ is the "effective" external magnetic field, and $\operatorname{sgn}\left(\mathrm{B}_{\mathrm{HF}}\right)$ is the sign of $\vec{B}_{\mathrm{HF}}$ with respect to $\vec{B}_{0}$. For the "effective" external magnetic field $B_{0}$ demagnetization effects have to be taken into account. For $0^{\circ}$ geometry a good approximation is given by

$$
\begin{gathered}
B_{0}=0 \text { for } B_{\text {ext }}<B_{\mathrm{dem}}, \\
B_{0}=B_{\mathrm{ext}}-B_{\mathrm{dem}} \text { for } B_{\mathrm{ext}}>B_{\mathrm{dem}},
\end{gathered}
$$

where the "demagnetization" field $B_{\text {dem }}$ depends on the ratio of thickness to linear dimensions of the actually used sample. (A typical value for the samples used in this work is $B_{\text {dem }}=0.5 \mathrm{kG}$.) For $90^{\circ}$ geometry Eqs. (4) can to a good approximation be replaced by

$$
B_{0}=B_{\mathrm{ext}}-\left[B_{\mathrm{dem}}\left(M_{z} / M_{z}^{\mathrm{sat}}\right)\right]
$$

where $M_{z}$ and $M_{z}^{\text {sat }}$ are the actual and the saturation magnetization, respectively. Here, the effective demagnetization 
field rises monotonically (almost linearly) from 0 to $B_{\mathrm{dem}}$ between $B_{\text {ext }}=0\left(B_{0}=0\right)$ and $B_{\text {ext }}=B_{\mathrm{A}}^{\perp}+B_{\text {dem }}\left(B_{0}=\right.$ $B_{\mathrm{A}}^{\perp}$.

The angular dependence of the hyperfine field (with respect to the $c$ axis) may be written as

$$
B_{\mathrm{HF}}(\theta)=B_{\mathrm{HF}}^{(\text {iso })}+B_{\mathrm{HF}}^{(\text {ani })} P_{2}(\cos \theta) .
$$

The connection between the isotropic and anisotropic contributions $B_{\mathrm{HF}}^{(\text {iso) }}$ and $B_{\mathrm{HF}}^{(\text {ani) }}$ with the "parallel", and "perpendicular', fields $B_{\mathrm{HF}}^{\|}$and $B_{\mathrm{HF}}^{\perp}$ is given by

$$
\begin{gathered}
B_{\mathrm{HF}}^{(\text {iso })}=1 / 3\left(B_{\mathrm{HF}}^{\|}+2 B_{\mathrm{HF}}^{\perp}\right), \\
B_{\mathrm{HF}}^{(\text {ani })}=2 / 3\left(B_{\mathrm{HF}}^{\|}-B_{\mathrm{HF}}^{\perp}\right) .
\end{gathered}
$$

\section{B. Quadrupole-interaction-resolved NMR-ON}

In ' $0{ }^{\circ}$ geometry,', the energy levels are given by

$$
E_{m}=-h \nu_{\mathrm{mag}}^{\|} m+h\left[\nu_{Q}^{(c)}+\nu_{Q}^{(L)}\right] \frac{3 m^{2}-I(I+1)}{4 I(2 I-1)} .
$$

For NMR-ON, there exists a set of $2 I$ subresonances. With the assumption that the $m=I$ state lies lowest in energy, the center of the subresonance corresponding to $\mathrm{rf}$ transitions between state $|m\rangle$ and $|m+1\rangle$ is given by

$$
\begin{gathered}
\nu_{m \rightarrow m+1}^{\|}=\nu_{\mathrm{mag}}^{\|}-\Delta \nu_{Q}^{\|}\left(m+\frac{1}{2}\right), \\
\Delta \nu_{Q}^{\|}=3\left[\nu_{Q}^{(c)}+\nu_{Q}^{(L)}\right] /[2 I(2 I-1)],
\end{gathered}
$$

where $\Delta \nu_{Q}^{\|}$is the $0^{\circ}$ subresonance separation.

In ' $90^{\circ}$ geometry," the energy levels must be calculated by diagonalization of Eq. (1). Let us, for a simplified discussion, first assume that the lattice quadrupole interaction is small compared to the magnetic interaction, i.e., $\left|\nu_{Q}^{(L)}\right| \ll$ $\nu_{\text {mag }}^{\perp}$. Then, first-order perturbation theory yields that the effective lattice quadrupole interaction follows a $P_{2}(\cos \theta)$ dependence ( $\theta$ is again the angle between the magnetization and the $c$ axis); i.e., for $\theta=90^{\circ}$,

$$
E_{m}=-h \nu_{\mathrm{mag}}^{\perp} m+h\left[\nu_{Q}^{(c)}-\frac{1}{2} \nu_{Q}^{(L)}\right] \frac{3 m^{2}-I(I+1)}{4 I(2 I-1)} .
$$

The set of NMR-ON resonance frequencies is now given by

$$
\begin{gathered}
\nu_{m \rightarrow m+1}^{\perp}=\nu_{\text {mag }}^{\perp}-\Delta \nu_{Q}^{\perp}\left(m+\frac{1}{2}\right), \\
\Delta \nu_{Q}^{\perp}=3\left[\nu_{Q}^{(c)}-\frac{1}{2} \nu_{Q}^{(L)}\right] /[2 I(2 I-1)],
\end{gathered}
$$

where $\Delta \nu_{Q}^{\perp}$ is the $90^{\circ}$ subresonance separation.

The difference of the (effective) quadrupole interaction frequency for $\theta=0^{\circ}$ and $90^{\circ}$ is given by $\nu_{Q}^{\|}-\nu_{Q}^{\perp}=\frac{3}{2} \nu_{Q}^{(L)}$; this is the basis for the resonance-offset technique described in Sec. II C.

In the NMR-ON method, the resonance absorption is detected via the change of the angular distribution of the emitted radiation. The angular distribution $W(\vartheta)$ and the anisotropy $A(\vartheta)$ of $\gamma$ rays emitted in the decay of oriented nuclei at the temperature $T$ are given by

$$
A(\vartheta)=W(\vartheta)-1=\sum_{k=2,4} A_{k} B_{k}\left(\nu_{M}, \nu_{Q}, T\right) P_{k}(\cos \vartheta) Q_{k}
$$

The parameters $A_{k}$ are products of the normally used angular correlation coefficients $U_{k}$ and $F_{k}$ which depend on the spins and the multipolarities of the decay cascade. The $P_{k}(\cos \vartheta)$ are Legendre polynomials, $\vartheta$ being the angle between the quantization axis (here the direction of the external magnetic field) and the direction of observation, and $Q_{k}$ are solid angle correction coefficients. The $B_{k}$ describe the degree of orientation; they depend on $\nu_{M}, \nu_{Q}$, and $T$. For hcp Co, $\nu_{M} \gg$ $\left|\nu_{Q}\right|$, the degree of orientation is mainly fixed by the ratio $\beta=h \nu_{M} / k_{B} T$ and nearly independent of $\nu_{Q}$.

Let us denote the subresonance corresponding to the energetically lowest sublevel transition as the $\nu^{(1)}$ resonance, the next $\nu^{(2)}, \ldots, i=1, \ldots, 2 I$. Normally, the $\nu^{(1)}$ subresonance has the largest subresonance amplitude; i.e., this resonance can normally be measured with the highest precision. The offset between $\nu^{(1)}$ and the magnetic interaction frequency is given by

$$
\nu^{(1)}=\nu_{\mathrm{mag}}-\left(I-\frac{1}{2}\right) \Delta \nu_{Q} .
$$

The resonance amplitude for the subresonance $i, \Delta A^{(i)}(\vartheta)$, is given by

$$
\Delta A^{(i)}(\vartheta)=A_{2} \Delta B_{2}^{(i)} P_{2}(\cos \vartheta) Q_{2}+A_{4} \Delta B_{4}^{(i)} P_{4}(\cos \vartheta) Q_{4},
$$

which can be calculated from the change of the orientation parameters $\Delta B_{k}^{(i)}$, as outlined in detail in Ref. [12]. For a precise theroretical prediction of $\Delta A^{(i)}(\vartheta)$, the field strength of the rf field at the nuclear site has to be known, which normally is not the case. In general, the amplitudes of the higher subresonances decrease continuously with $i$, as the corresponding sublevel population probabilities decrease with the Boltzmann factor $e^{-\beta}$, depending on the temperature $T$. Additionally, the subresonance amplitudes depend on the ratio $A_{4} / A_{2}$. For the case $A_{4}=0$, e.g., for pure $E 1$ or $M 1$ transitions, the relative subresonance amplitudes are the same for $\vartheta=0^{\circ}$ and $90^{\circ}$. For $A_{4} / A_{2} \neq 0$ the relative subresonance amplitudes are different for $\vartheta=0^{\circ}$ and $90^{\circ}$. (This is the basis for the so-called $0^{\circ}-90^{\circ}$ resonance displacement technique [12]; see also below.) In the special case of stretched $E 2$ transitions, $\Delta A^{(1)}\left(90^{\circ}\right) \rightarrow 0$ for $T \rightarrow 0$; i.e., the $\nu^{(1)}$ resonance is unobservable for $\vartheta=90^{\circ}$ at low temperatures. For mixed-multipolarity transitions the relative subresonance amplitudes depend on the mixing ratio $\delta$. The maximum sensitivity for the determination of the subresonance separation and hence the quadrupole splitting is obtained at intermediate temperatures: At very low temperatures the amplitude of the $\nu^{(1)}$ resonance is large; however, only a few subresonances can be observed. At higher temperatures more subresonances can be observed, the amplitudes becoming smaller because of the smaller $\gamma$ anisotropy.

For $90^{\circ}$ geometry, and for the case that the condition $\left|\nu_{Q}\right| \ll \nu_{M}$ is not fulfilled, the energy eigenvalues must be calculated by explicit diagonalization. Then, it is found that the subresonance separation is no longer constant but depends on the sublevel transition. To faciliate the least- 
squares fit of measured $90^{\circ}$ resonance spectra, this effect is taken into account by diagonalizing the Hamiltonian and calculating correction coefficients $K^{(i)}$ for the subresonance $i$,

$$
\Delta \nu_{Q}^{(i)}=K^{(i)} \Delta \nu_{Q}
$$

and

$$
\nu^{(1)}=\nu_{\mathrm{mag}}-K^{(\mathrm{mag})}\left(I-\frac{1}{2}\right) \Delta \nu_{Q} .
$$

Within the framework of this description, $K^{(i)}=K^{(\mathrm{mag})}=1$ for $\Delta \nu_{Q}^{(\mathrm{c})}=0$ and $\left|\nu_{Q}\right| \ll \nu_{M}$.

\section{Resonance-offset method}

With increasing spin, according to Eq. (9), the quadrupole subresonance separation becomes smaller, and there exists a critical spin value $I_{c}$ above which the quadrupole substructure cannot be resolved. The critical spin values depend on several parameters, such as the ratio of the quadrupole splitting to the magnetic splitting and the inhomogeneous linewidth. For the case that the quadrupole splitting cannot be resolved, a broadened (and possibly asymmetric) NMR-ON resonance is expected. The resonance center is then given by

$$
\bar{\nu}=\nu_{M}-\Delta \nu_{Q}\left(\bar{m}+\frac{1}{2}\right),
$$

where $\left(\bar{m}+\frac{1}{2}\right)$ is given by

$$
\left(\bar{m}+\frac{1}{2}\right)=\sum_{i=1}^{2 I}\left(m^{(i)}+\frac{1}{2}\right) \Delta A^{(i)}(\vartheta) / \sum_{i=1}^{2 I} \Delta A^{(i)}(\vartheta) .
$$

Equation (20) shows that, for an independent determination of $\nu_{M}$ and $\nu_{Q}$, two different measurements are necessary, for which the functional dependence on $\nu_{M}$ and $\nu_{Q}$ is different. In Ref. [12] it has been shown that $\left(\bar{m}+\frac{1}{2}\right)$ may be slightly different for $\vartheta=0^{\circ}$ and $90^{\circ}$, where $\vartheta$ is the angle of $o b$ servation with respect to the quantization axis. This is due to the fact that

$$
\begin{gathered}
\Delta A^{(i)}\left(0^{\circ}\right) \sim A_{2} \Delta B_{2}^{(i)} Q_{2}+A_{4} \Delta B_{4}^{(i)} Q_{4}, \\
\Delta A^{(i)}\left(90^{\circ}\right) \sim-\frac{1}{2} A_{2} \Delta B_{2}^{(i)} Q_{2}+\frac{3}{8} A_{4} \Delta B_{4}^{(i)} Q_{4},
\end{gathered}
$$

which are different for $A_{4} / A_{2} \neq 0$.

The maximum difference of the resonance centers for $\vartheta$ $=0^{\circ}$ and $90^{\circ}$ is, however, only a fraction of $\Delta \nu_{Q}^{\text {eff }}$, i.e., very small, which has the consequence that this method is limited to few selected systems which allow the determination of the resonance frequencies with extremely high precision. If, however, the resonance is measured for $\theta=0^{\circ}$ and $90^{\circ}$, i.e., magnetization parallel and perpendicular to the single-crystal $c$ axis, the resonance centers are given by

$$
\begin{gathered}
\bar{\nu}^{\|}=\nu_{M}^{\|}-\left[\Delta \nu_{Q}^{(c)}+\Delta \nu_{Q}^{(L)}\right]\left(\bar{m}+\frac{1}{2}\right), \\
\bar{\nu}^{\perp}=\nu_{M}^{\perp}-\left[\Delta \nu_{Q}^{(c)}-\frac{1}{2} \Delta \nu_{Q}^{(L)}\right]\left(\bar{m}+\frac{1}{2}\right) .
\end{gathered}
$$

Assuming that $\left(\bar{m}+\frac{1}{2}\right)$ is identical for $0^{\circ}$ and $90^{\circ}$, the resonance offset is now given by

$$
\overline{\Delta \nu}=\Delta \nu_{\mathrm{mag}}-\frac{3}{2}\left(\bar{m}+\frac{1}{2}\right) \Delta \nu_{Q}^{(L)},
$$

where the magnetic contribution to the offset is given by

$$
\Delta \nu_{\mathrm{mag}}=g \mu_{N}\left[\left(B_{\mathrm{HF}}^{\|}-B_{\mathrm{HF}}^{\perp}\right)+\operatorname{sgn}\left(B_{\mathrm{HF}}\right)(1+K)\left(B_{0}^{\|}-B_{0}^{\perp}\right)\right] / h,
$$

which can be predicted with high precision, as $B_{\mathrm{HF}}^{\|}$and $B_{\mathrm{HF}}^{\perp}$ can be determined from quadrupole-interaction-resolved spectroscopy as described in Sec. II B. Here $B_{0}^{\|}$and $B_{0}^{\perp}$ are the external magnetic fields at which the measurements parallel and perpendicular to the $c$ axis are performed, which may be chosen to be equal. As $\left(\bar{m}+\frac{1}{2}\right)$ is of the order of $I$ for high-spin states, the so-called "parallel-perpendicular resonance offset" is so large that it can easily be measured. The only uncertainty for the determination of the quadrupole moment is then given by the (theoretical) uncertainty of $(\bar{m}+$ $\frac{1}{2}$ ). This uncertainty is mainly due to the dependence of the relative subresonance amplitudes on the rf power at the nuclear site, which is known only with limited accuracy. However, as $\left(\bar{m}+\frac{1}{2}\right)$ depends on the temperature at which the resonances are measured, a measurement of the resonance centers as function of the temperature and as function of the rf power may yield redundant information. Thus, in comparison to the $0^{\circ}-90^{\circ}$ resonance displacement, which is only a fraction of $\Delta \nu_{Q}$, the "parallel-perpendicular resonance offset' 'is $\frac{3}{2}\left(\bar{m}+\frac{1}{2}\right) \Delta \nu_{Q}^{(L)}$, i.e., much larger and, hence, much easier to measure. In addition, it is also present for $\gamma$ transitions with $A_{4}=0$, for which the $0^{\circ}-90^{\circ}$ resonancedisplacement method is not applicable.

\section{EXPERIMENTAL DETAILS}

All samples were prepared by mass-separator implantation at ISOLDE/CERN. The hcp Co single-crystal disks were prepared in the following way: Disks with a diameter of $\approx 10 \mathrm{~mm}$ and a thickness of $\approx 0.2 \mathrm{~mm}$ were spark cut from a bulk hcp Co single-crystal available commercially. The orientation of the $c$ axis was chosen to be parallel to the plane of the disk. With Laue backscattering techniques an accuracy of $<1^{\circ}$ was achieved for the alignment of the $c$ axis. The further treatment consisted of many steps as described in detail in Ref. [13]: (i) mechanical polishing with 15- $\mu \mathrm{m}, 9-\mu \mathrm{m}$, and 3- $\mu \mathrm{m}$ "diamond disks," (ii) mechanical polishing with $1-\mu \mathrm{m}, 0.5-\mu \mathrm{m}$, and $0.25-\mu \mathrm{m}$ diamond paste (after each mechanical polishing step the crystal was cleaned ultrasonically and polished chemically), and (iii) electropolishing $(\sim 6 \mathrm{~min})$ in $\mathrm{H}_{3} \mathrm{PO}_{4}(85 \%)$ with an abrasion rate of $\approx 0.1 \mu \mathrm{m} / \mathrm{min}$. In this way it could be assured that the quality of the single-crystal structure was not deteriorated in the surface layer in which the radioactive isotopes are deposited by the implantation. The Lu isotopes were obtained by the ${ }^{181} \mathrm{Ta}(p, x n y p)$ spallation reaction at CERN. With the on-line mass separator ISOLDE the Lu isotopes were implanted into the hcp Co single-crystal disks with an implantation voltage of $60 \mathrm{kV}$. The size of the implantation area was restricted to a spot of $\sim 3 \mathrm{~mm}$ in diameter at the center of the crystals. Typical implantation times were between 10 $\min \left({ }^{169} \mathrm{Lu}\right)$ and $2 \mathrm{~h}\left({ }^{173} \mathrm{Lu}\right)$. In all cases the doses were less than $10^{14} \mathrm{~cm}^{-2}$.

The samples were soldered with In to the $\mathrm{Cu}$ coldfinger of 
a ${ }^{3} \mathrm{He}-{ }^{4} \mathrm{He}$-dilution refrigerator (Oxford Instruments, model TL-400) with top-loading facility. For the alignment of the single-crystal $c$ axis with respect to the magnetic field in the measuring position of the cryostat, a typical accuracy of $\sim 1^{\circ}$ was achieved. The samples were cooled to temperatures $T \approx 10 \mathrm{mK}$ and measurements were performed for $B_{0} \| c$ (" $00^{\circ}$ geometry") and $B_{0} \perp c$ (" $90^{\circ}$ geometry"). For the case $B_{0} \| c$ only a small external magnetic field $B_{\text {ext }}=$ 0.5-1 kG was applied to ensure the soldered joint to be normal conducting. For the case $B_{0} \perp c$ the external magnetic field was chosen $B_{\text {ext }}=20 \mathrm{kG}$; in this way, it was assured that the magnetization could be forced parallel to $B_{0}$.

The $\gamma$ rays were detected with two $\mathrm{Ge}(\mathrm{Li})$ detectors (efficiency $\approx 20 \%$ ) and two $p$-type intrinsic Ge detectors (efficiency $\approx 40 \%$ ) placed at $0^{\circ}, 90^{\circ}, 180^{\circ}$, and $270^{\circ}$ with respect to the external magnetic field $B_{\text {ext }}$.

For the NMR-ON experiments, the radio frequency was applied either with a one-turn or a double-turn Helmholtztype rf coil, the "free" coil always being used to monitor the rf power. The rf was supplied by a voltage-tuned rf generator (Rohde \& Schwarz, type SMLU) the center frequency and modulation bandwidth being continuously controlled by a feedback system. In comparison to the normally used radio frequency synthesizers it has the advantage of ultrabroadband frequency modulation, which was decisive for the measurements on ${ }^{177 m} \mathrm{Lu}$. The modulation frequency was chosen $100 \mathrm{~Hz}$; the frequency modulation bandwidth was varied between $\pm 2 \mathrm{MHz}$ and $\pm 5 \mathrm{MHz}$. The center frequency was varied in steps over the resonance region. Because of the large quadrupole splitting, the frequency regions of interest were anomalously large, which has an important implication for the NMR-ON experiments: Because of the unmatched coupling between the rf generator and the rf coils, the rf field strength at the sample site depends on the frequency. This has the consequences that (i) the rf-induced NMR transition probability and (ii) the nonresonant rf eddy current heating depend on the frequency of the applied rf field. As a consequence of this frequency dependence, resonance structures occur in the $\gamma$-anisotropy spectrum, which are generally much broader than typical NMR-ON resonances with $\mathrm{Fe}, \mathrm{Ni}$, or fcc $\mathrm{Co}$ as host matrices. Thus, within small frequency regions, the applied rf power normally does not vary too strongly, and the absolute value of the rf power at the sample site can be chosen by the appropriate rf power of the $\mathrm{rf}$ generator. If the width of the NMR-ON structure becomes comparable to the width of resonances of the rf system, special care has to be taken to separate the NMR-ON signal from the spurious resonances caused by the eddy-current heating which is resonant with respect the rf system but nonresonant with respect to the nuclear spin system. Two different approaches have been pursued in the present work to overcome this experimental problem.

(i) Successive measurements with and without freqency modulation (FM). Here the idea is that the nonresonant eddycurrent heating is monitored by measuring without FM. Thus, subtracting successive spectra measured with and without FM should yield the "true" resonance spectra. For systems with broad resonances and small resonance effects this procedure is not sufficient, because of the nonlinearity of the frequency dependence of the eddy-current heating, which becomes important for large frequency modulation bandwidths, the latter being necessary if the linewidth is large. In the present work this effect has been taken into account by interpolation of the nonresonant background according to the frequency modulation bandwidth with respect to the frequency steps. However, in this way only static effects are compensated. There is, however, also a dynamic temperature variation caused by the frequency-dependent eddy-current heating, together with the large heat capacity of the Co single crystals (caused by the Schottky anomaly of the specific heat due to the magnetic interaction of stable ${ }^{59} \mathrm{Co}$ in hcp Co). To compensate these effects to first order, the measurements at a fixed frequency were performed with the following sequence: FM-on, FM-off, FM-off, FM-on.

(ii) At every frequency, the rf power was adjusted in such a way that the average eddy-current heating remained constant in the whole frequency region. This was performed by measuring "power tables" for the frequency regions of interest with an iterative procedure: First, for the frequency region of interest, the temperature of the samples was measured as a function of the frequency of the applied rf field with constant power. Then, at several characteristic frequencies, the temperature of the samples was measured as function of the applied rf power. Next, a power table for this region was generated and the temperature as function of the frequency was measured again. This procedure was repeated iteratively, until a flat dependence of the temperature as function of the frequency was obtained. In most cases, two or three iterations were sufficient. Although probably not necessary, even with the rf-power adjustment, the NMR-ON measurements were performed with the FM sequence "onoff-off-on."

\section{RESULTS}

\section{A. ${ }^{177} \mathrm{LuCo}(\mathrm{hcp})$}

There are two strong $\gamma$ transitions in the decay of $6.7 \mathrm{~d}$ $7 / 2+{ }^{177} \mathrm{Lu}$ suitable for NMR-ON.

(i) $9 / 2^{+}(321 \mathrm{keV}) \rightarrow 9 / 2^{-}(113 \mathrm{keV})$ :

$E_{\gamma}=208 \mathrm{keV}, I_{\gamma}=11 \%, E 1+M 2, \quad \delta(E 2 / M 1) \lesssim 0.08 ;$

(ii) $9 / 2^{-}(113 \mathrm{keV}) \rightarrow 7 / 2^{-}(0 \mathrm{keV})$ :

$$
\begin{gathered}
E_{\gamma}=113 \mathrm{keV}, \quad I_{\gamma}=6.6 \%, \\
E 2+M 1 \quad \delta(E 2 / M 1) \sim-4 .
\end{gathered}
$$

The NMR-ON spectra measured in $0^{\circ}$ geometry at $T=$ $17 \mathrm{mK}$ are shown in Fig. $1(113 \mathrm{keV})$ and Fig. $2(208 \mathrm{keV})$, in both cases for $\vartheta=0^{\circ}$ (top) and $\vartheta=90^{\circ}$ (bottom). The strong suppression of the $\nu_{1}$ resonance at $\vartheta=90^{\circ}$ for the $113 \mathrm{keV} \gamma$ transition is due to the strong $E 2$ character of this transition. The solid lines are the results of least-squares fits of five equidistant lines with Gaussian shape and linewidth $\Gamma$. The parameters are $\nu_{1}, \Delta \nu_{Q}, \Gamma, \Delta A^{(i)}\left(0^{\circ}\right)$, and $\Delta A^{(i)}\left(90^{\circ}\right), i=1, \ldots, 5$. After correction for the external magnetic field we adopt as final results

$$
\nu_{M}\left({ }^{177} \mathrm{LuCo}\right)=249.28(17) \mathrm{MHz},
$$




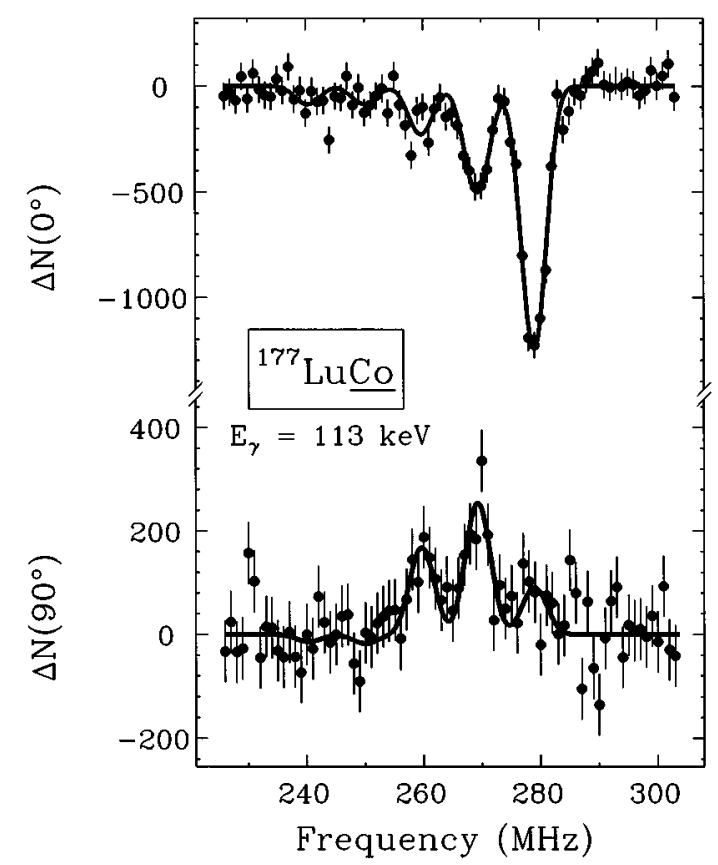

FIG. 1. Quadrupole-interaction-resolved (QI) NMR-ON resonance of the $113 \mathrm{keV}$ transition of ${ }^{177} \mathrm{Lu}$. Top: $\vartheta=0^{\circ}, \gamma$ emission $\| B_{\text {ext }}$. Bottom: $\vartheta=90^{\circ}, \gamma$ emission $\perp B_{\text {ext }}$. The $\nu_{1}$ resonance is strongly suppressed for $\vartheta=90^{\circ}$ because of the dominant $E 2$ character of the $\gamma$ transition.

$$
\nu_{Q}\left({ }^{177} \mathrm{LuCo}\right)=-139.96(90) \mathrm{MHz} .
$$

Taking $\mu\left({ }^{177} \mathrm{Lu}\right)=2.239(11) \mu_{n}$ and $Q\left({ }^{177} \mathrm{Lu}\right)=3.39(2) \mathrm{b}$ [14], the magnetic hyperfine field and the electric field gradient are then found to be

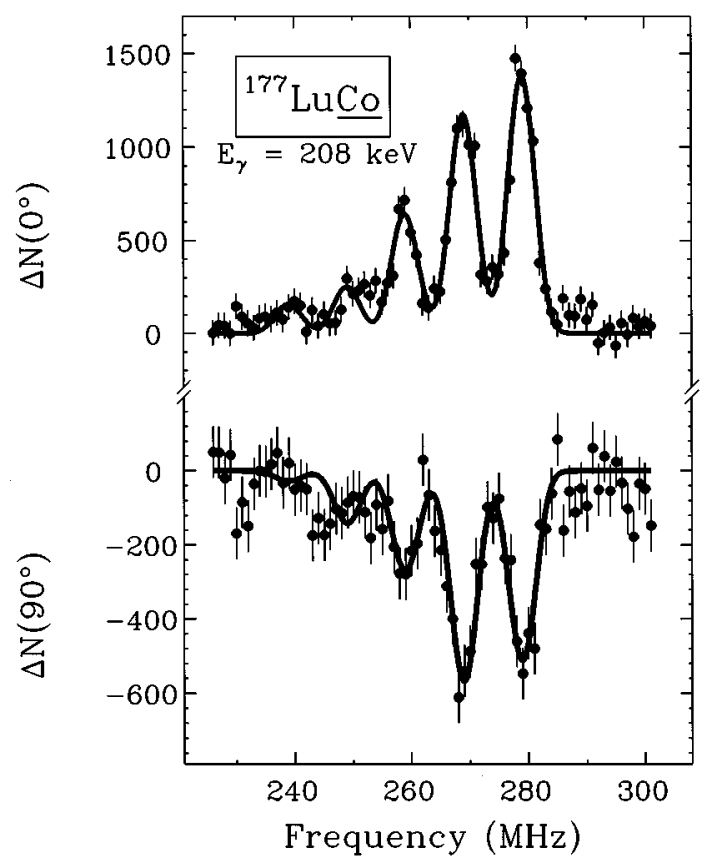

FIG. 2. QI-NMR-ON resonance of the $208 \mathrm{keV}$ transition of ${ }^{177}$ Lu. Top: $\vartheta=0^{\circ}, \gamma$ emission $\| B_{\text {ext }}$. Bottom. $\vartheta=90^{\circ}, \gamma$ emission $\perp B_{\text {ext }}$. In contrast to the $113 \mathrm{keV}$ transition (Fig. 1), the $\nu_{1}$ resonance is much less suppressed for $\vartheta=90^{\circ}$.

$$
\begin{gathered}
B_{\mathrm{HF}}^{\|}=-511.2(2.5) \mathrm{kG}, \\
e q^{\|}=-1.707(15) \times 10^{17} \mathrm{~V} / \mathrm{cm}^{2} .
\end{gathered}
$$

\section{B. ${ }^{169} \mathrm{LuCo}(\mathrm{hcp})$}

In the decay of ${ }^{169} \mathrm{Lu}\left(I^{\pi}=7 / 2^{+}, \mathrm{T}_{1 / 2}=34.1 \mathrm{~h}\right)$ the $\gamma$ transitions with $191 \mathrm{keV}\left(I_{\gamma}=22 \%\right), 960 \mathrm{keV}\left(I_{\gamma}=24 \%\right)$, and $1450 \mathrm{keV}\left(I_{\gamma}=9 \%\right)$ are sufficiently strong for NMR-ON experiments. A $0^{\circ}$ geometry NMR-ON spectrum of the $960 \mathrm{keV}$ transition measured with $B_{\mathrm{ext}}=1 \mathrm{kG}$ is illustrated in Fig. 3 (top). Here two experiments with two different implantations and different single crystals were performed. They yielded slightly different results. The origin for this difference could not be found. Therefore the errors quoted below include this uncertainty. Taking into account the data for the 191 and $1450 \mathrm{keV}$ transitions and correcting for the external magnetic field, the final results for the magnetic and electric quadrupole interaction frequencies are

$$
\begin{gathered}
\nu_{M}=255.7(7) \mathrm{MHz}, \\
\nu_{Q}=-141.4(5.0) \mathrm{MHz} .
\end{gathered}
$$

Taking into account the hyperfine splitting frequencies of ${ }^{177} \mathrm{LuCo}(\mathrm{hcp})$, the following ratios are deduced:

$$
\begin{gathered}
\mu\left({ }^{169} \mathrm{Lu}\right) / \mu\left({ }^{177} \mathrm{Lu}\right)=1.026(3), \\
Q\left({ }^{169} \mathrm{Lu}\right) / Q\left({ }^{177} \mathrm{Lu}\right)=+1.01(4) .
\end{gathered}
$$

The nuclear moments are

$$
\begin{aligned}
& \mu\left({ }^{169} \mathrm{Lu}\right)=2.297(13) \mu_{N}, \\
& Q\left({ }^{169} \mathrm{Lu}\right)=+3.42(12) \quad \text { b. }
\end{aligned}
$$

Additionally, a $90^{\circ}$ geometry measurement was performed, with an external magnetic field $B_{\text {ext }}=20 \mathrm{kG}$. The result is shown in the bottom part of Fig. 3. Because of the relatively large quadrupole splitting (with respect to the magnetic interaction), the subresonances are not separated equidistantly and the correction factors $K^{(i)}$ and $K^{\mathrm{mag}}$ as defined by Eqs. (18) and (19) have to be taken into account for the least-squares fit. These were obtained by an iterative procedure, determination of $\nu_{M}$ and $\nu_{Q}$ and subsequent calculation of $K^{(i)}$ and $K^{(\mathrm{mag})}$, with which then a new set of $\nu_{M}$ and $\nu_{O}$ could be determined. The final correction factors were $K^{(1)}=1.079, \quad K^{(2)}=1.043, \quad K^{(3)}=1.011, \quad K^{(4)}=0.982$, $K^{(5)}=0.955, K^{(6)}=0.931$, and $K^{(\mathrm{mag})}=1.022$.

The results from the least-squares fit are

$$
\begin{gathered}
\nu_{M}^{(\perp)}(20 \mathrm{kG})=234.8(3) \mathrm{MHz}, \\
\nu_{Q}^{(\perp)}=-148.4(3.1) \mathrm{MHz} .
\end{gathered}
$$

After correction for the external magnetic field, the final result for the magnetic hyperfine splitting frequencies is

$$
\nu_{M}^{(\perp)}=244.3(4) \mathrm{MHz} .
$$

The quadrupole interaction frequency is, within the experimental uncertainty, the same as in the $0^{\circ}$ geometry. This 


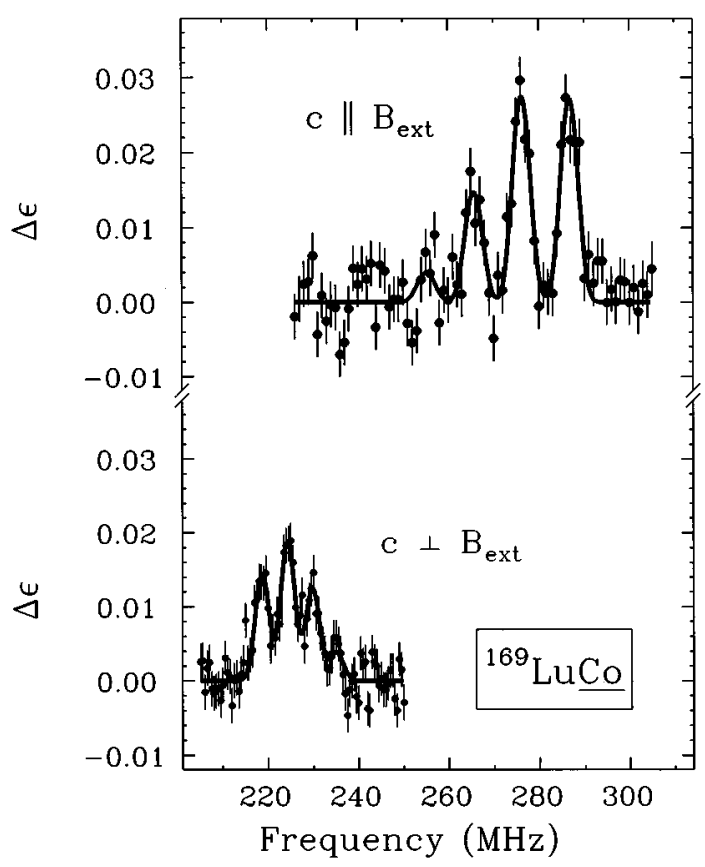

FIG. 3. Change of the $\gamma$ anisotropy $\Delta \epsilon$ of the $960 \mathrm{keV}$ transition of ${ }^{169} \mathrm{Lu}\left[\epsilon=W\left(\vartheta=0^{\circ}\right) / W\left(\vartheta=90^{\circ}\right)-1\right]$. Top: $0^{\circ}$ geometry, $c \| B_{\text {ext }}, B_{\text {ext }}=1 \mathrm{kG}$. Bottom: $90^{\circ}$ geometry, $c \perp B_{\text {ext }}, B_{\text {ext }}=20$ $\mathrm{kG}$.

means that there is no evidence for the existence of a "collinear" EFG. The "perpendicular' magnetic hyperfine field is found to be

$$
B_{\mathrm{HF}}^{\perp}=-489.4(2.6) \mathrm{kG} \text {, }
$$

which is $\sim 4 \%$ smaller in magnitude than $B_{\mathrm{HF}}^{\|}$.

\section{C. ${ }^{171} \mathrm{LuCo}(\mathrm{hcp})$}

There is one strong $\gamma$ transition in the decay of ${ }^{171} \mathrm{Lu}$ $\left(E=740 \mathrm{keV}, I_{\gamma}=53 \%\right)$ the $\gamma$ anisotropy of which is sufficiently large for NMR-ON. The resonance structure was measured in $0^{\circ}$ geometry, first, for an external magnetic field $B_{\text {ext }}=1 \mathrm{kG}$; at three different temperatures, $T=12,22$, and $43 \mathrm{mK}$. Additionally, for $T=12 \mathrm{mK}$, measurements were performed for an external magnetic field $B_{\text {ext }}=6 \mathrm{kG}$; Fig. 4

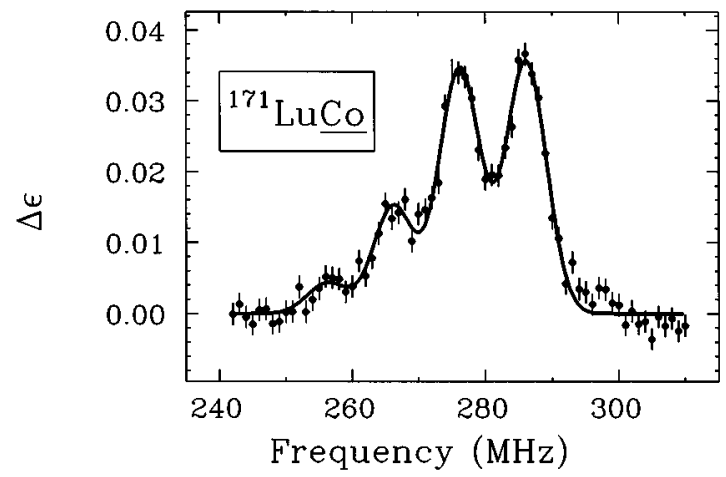

FIG. 4. QI-NMR-ON resonance for the $740 \mathrm{keV}$ transition of ${ }^{171} \mathrm{Lu}$. The linewidth is slightly larger than for ${ }^{177} \mathrm{Lu}$ (Figs. 1 and 2) ${ }^{169} \mathrm{Lu}$ (Fig. 3), and ${ }^{173} \mathrm{Lu}$ (Fig. 6). shows the result for the $12 \mathrm{mK}$ measurement with $B_{\text {ext }}=1$ $\mathrm{kG}$. The measurements showed that, as expected, there is no temperature dependence of the hyperfine splitting frequencies. The shift of $\nu_{M}$ with $B_{\text {ext }}$ was found to be $-0.47(16)$ $\mathrm{MHz} / \mathrm{kG}$, in good agreement with -0.501(2) $\mathrm{MHz} / \mathrm{kG}$, expected with the known $g$ factor and assuming $K=0$ for the Knight shift parameter. The dependence of the quadrupole interaction frequency on the external magnetic field is $d \nu_{Q} / d B_{\text {ext }}=-0.30(64) \mathrm{MHz} / \mathrm{kG}$, i.e., zero within the statistical accuracy. On the other hand, as a function of $B_{\text {ext }}$, the best accuracy is obtained for the $\nu_{1}$ resonance. Assuming that the quadrupole interaction frequency is independent of $B_{\text {ext }}$, the shift of $\nu_{1}$ should be the same as the shift of $\nu_{M}$. The result is $d \nu_{1} / d B_{\text {ext }}=-0.40(4) \mathrm{MHz} / \mathrm{kG}$, which is significantly smaller than $-0.501(2) \mathrm{MHz} / \mathrm{kG}$. If we ascribe this difference to a magnetic field dependence of the quadrupole interaction, we derive $d \nu_{Q} / d B_{\text {ext }}=-0.47(19) \mathrm{MHz} /$ $\mathrm{kG}$. Obvioulsy, there is actually a (weak) dependence of the quadrupole interaction on $B_{\text {ext }}$, which, however, should not be overinterpreted. Therefore, for the derivation of ratios of quadrupole moments, we always take the quadrupole interaction frequencies measured for $B_{\text {ext }}=1 \mathrm{kG}$. As final results for the magnetic and electric quadrupole interaction frequencies we adopt

$$
\begin{gathered}
\nu_{M}=256.6(3) \mathrm{MHz}, \\
\nu_{Q}=-139.5(9) \mathrm{MHz} .
\end{gathered}
$$

The following ratios are deduced:

$$
\begin{aligned}
& \mu\left({ }^{171} \mathrm{Lu}\right) / \mu\left({ }^{177} \mathrm{Lu}\right)=1.0294(14), \\
& Q\left({ }^{171} \mathrm{Lu}\right) / Q\left({ }^{177} \mathrm{Lu}\right)=+0.997(9) .
\end{aligned}
$$

The nuclear moments are

$$
\begin{gathered}
\mu\left({ }^{171} \mathrm{Lu}\right)=2.305(12) \mu_{N}, \\
Q\left({ }^{171} \mathrm{Lu}\right)=+3.38(4) \text { b. } \\
\text { D. }{ }^{172} \mathbf{L u C o}(\text { hcp })
\end{gathered}
$$

In the decay of $6.7 \mathrm{~d}{ }^{172} \mathrm{Lu}\left(I^{\pi}=4^{-}\right)$the following $\gamma$ transitions have sufficiently large $\gamma$ anisotropies: $E_{\gamma}=91$ $\mathrm{keV}, I_{\gamma}=4.9 \% ; E_{\gamma}=181 \mathrm{keV}, I_{\gamma}=10.2 \% ; E_{\gamma}=810$ $\mathrm{keV}, I_{\gamma}=15.3 \% ; E_{\gamma}=912 \mathrm{keV}, I_{\gamma}=14.2 \%$; and $E_{\gamma}=$ $1093 \mathrm{keV}, I_{\gamma}=75.2 \%$. Figure 5 shows the NMR-ON spectrum of the $1093 \mathrm{keV}$ transition. The quadrupole subresonance separation $\Delta \nu_{Q}$ was consistent for all $\gamma$ transitions, with results $-8.12(35),-8.41(18),-8.84(47),-8.32(32)$, and $-8.39(15) \mathrm{MeV}$, respectively. The final results are

$$
\begin{gathered}
\nu_{M}=281.9(3) \mathrm{MHz}, \\
\nu_{Q}=-156.6(1.9) \mathrm{MHz},
\end{gathered}
$$

and the ratios

$$
\begin{gathered}
\mu\left({ }^{172} \mathrm{Lu}\right) / \mu\left({ }^{177} \mathrm{Lu}\right)=1.292(2), \\
Q\left({ }^{172} \mathrm{Lu}\right) / Q\left({ }^{177} \mathrm{Lu}\right)=+1.12(2)
\end{gathered}
$$




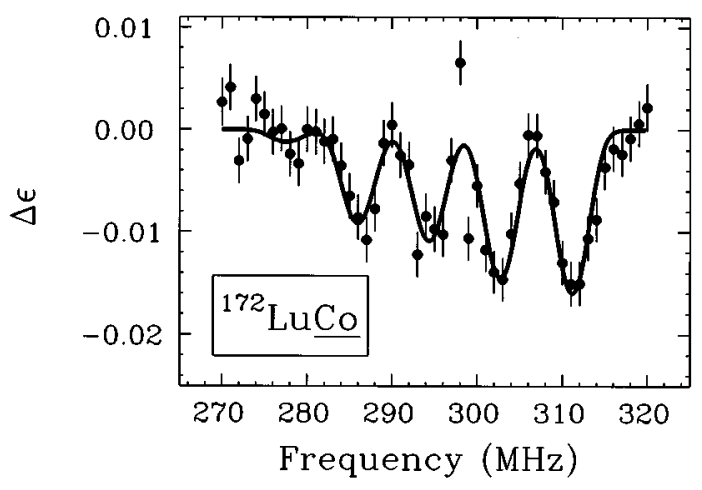

FIG. 5. QI-NMR-ON resonance for the $1093 \mathrm{keV}$ transition of ${ }^{172} \mathrm{Lu}$.

The nuclear moments are

$$
\begin{gathered}
\mu\left({ }^{172} \mathrm{Lu}\right)=2.893(15) \mu_{N}, \\
Q\left({ }^{172} \mathrm{Lu}\right)=+3.79(6) \quad \mathrm{b} . \\
\text { E. }{ }^{173} \mathbf{L u C o}(\text { hcp })
\end{gathered}
$$

Because of the relatively long half-life of ${ }^{173} \mathrm{Lu}\left(T_{1 / 2}=\right.$ $1.4 \mathrm{yr}, I^{\pi}=7 / 2^{+}$), the sample was weak, with the implication of long measurement times. The three strongest $\gamma$ transitions with $E_{\gamma}=79 \mathrm{keV}\left(I_{\gamma}=7.5 \%\right), E_{\gamma}=100 \mathrm{keV}\left(I_{\gamma}=\right.$ $3.1 \%)$, and $E_{\gamma}=272 \mathrm{keV}\left(I_{\gamma}=12.5 \%\right)$ had sufficiently large $\gamma$ anisotropies for NMR-ON. Here, in addition to the standard NMR-ON measurement technique, measurements were performed in which the rf power for each frequency was adjusted so that the frequency dependence of the eddycurrent heating was compensated. A typical spectrum for the $272 \mathrm{keV}$ transition is shown in Fig. 6. The results without power adjustment are

$$
\begin{gathered}
\nu_{M}=253.9(3) \mathrm{MHz}, \\
\nu_{Q}=-147.8(1.4) \mathrm{MHz},
\end{gathered}
$$

and with power adjustment,

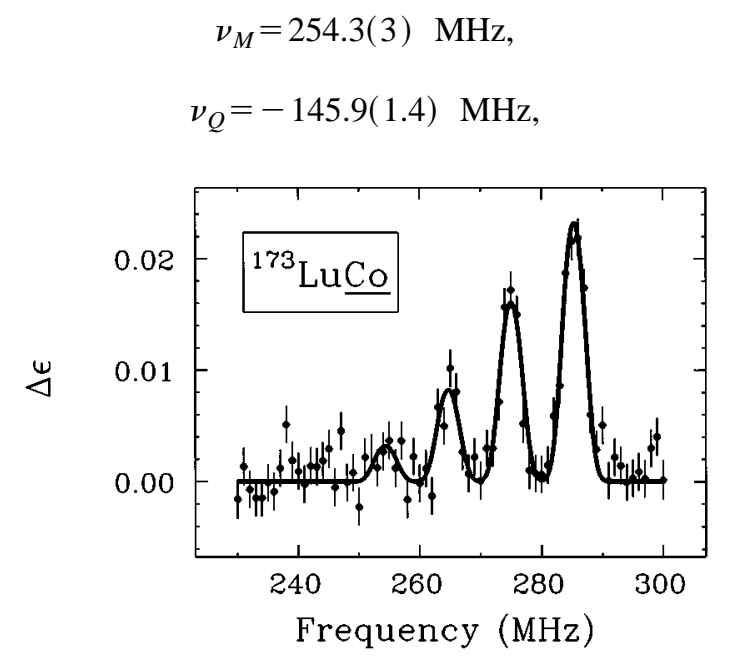

FIG. 6. QI-NMR-ON resonance for the $272 \mathrm{keV}$ transition of ${ }^{173} \mathrm{Lu}$.

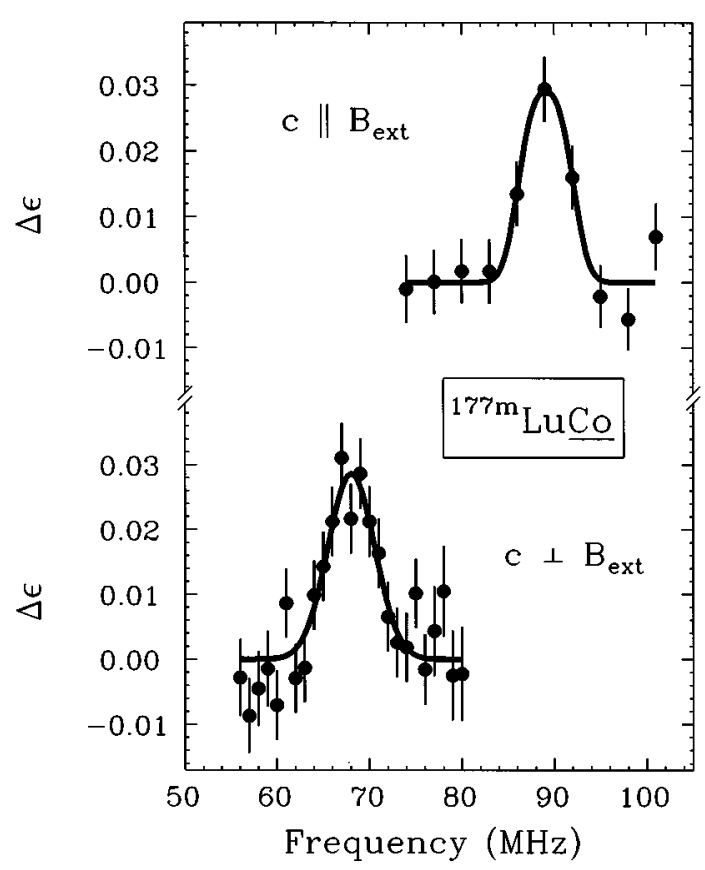

FIG. 7. Unresolved NMR-ON spectra for ${ }^{177 m} \mathrm{Lu}$. Top: $0^{\circ}$ geometry, $c \| B_{\text {ext }}, B_{\text {ext }}=1 \mathrm{kG}$. Bottom: $90^{\circ}$ geometry, $c \perp B_{\text {ext }}$, $B_{\text {ext }}=20 \mathrm{kG}$.

They agree within the statistical error. As final results we adopt the average

$$
\begin{gathered}
\nu_{\mathrm{M}}=254.1(3) \mathrm{MHz}, \\
\nu_{Q}=-146.9(1.0) \mathrm{MHz} .
\end{gathered}
$$

For the nuclear moments we get

$$
\begin{gathered}
\mu\left({ }^{173} \mathrm{Lu}\right) / \mu\left({ }^{177} \mathrm{Lu}\right)=1.0185(14), \\
Q\left({ }^{173} \mathrm{Lu}\right) / Q\left({ }^{177} \mathrm{Lu}\right)=+1.05(1) .
\end{gathered}
$$

The nuclear moments are

$$
\begin{gathered}
\mu\left({ }^{173} \mathrm{Lu}\right)=2.280(12) \mu_{N}, \\
Q\left({ }^{173} \mathrm{Lu}\right)=+3.56(4) \text { b. } \\
\text { F. }{ }^{177 \mathrm{~m}} \mathbf{L u C o ( h c p )}
\end{gathered}
$$

Because of the high spin of ${ }^{177 \mathrm{~m}} \mathrm{Lu}\left(T_{1 / 2}=162 \mathrm{~d}, I^{\pi}=\right.$ $23 / 2^{-}$) it was not possible to resolve the quadrupole substructure. Therefore the "parallel-perpendicular resonanceoffset" method described in Sec. II C was used to determine the nuclear moments. NMR-ON measurements were performed for $B_{\text {ext }} \| c\left(B_{\text {ext }}=1 \mathrm{kG}\right)$ and $B_{\text {ext }} \perp c\left(B_{\text {ext }}=20\right.$ $\mathrm{kG})$. Figure 7 shows the NMR-ON spectra of the $418 \mathrm{keV}$ transition. Taking into account the results of all other transitions with sufficient intensity, the final results are

$$
\begin{gathered}
\nu^{(\|)}\left(B_{\text {ext }}=1 \quad \mathrm{kG}\right)=88.4(3) \mathrm{MHz}, \\
\nu^{(\perp)}\left(B_{\text {ext }}=20 \mathrm{kG}\right)=68.1(2) \mathrm{MHz} .
\end{gathered}
$$


TABLE I. Magnetic moments of $\mathrm{Lu}$ isotopes of this work (NMR-ON), laser spectroscopy (LS) of Ref. [10], and Nuclear Orientation (NO).

\begin{tabular}{|c|c|c|c|c|c|}
\hline \multirow[t]{2}{*}{ Isotope } & \multirow[t]{2}{*}{$I^{\pi}$} & \multicolumn{2}{|c|}{$\mu\left[\mu_{N}\right]$} & \multicolumn{2}{|c|}{$\mu^{(\mathrm{NMR}-\mathrm{ON})} / \mu^{(\mathrm{LS})}$} \\
\hline & & $\mathrm{NMR}^{-O N}{ }^{\mathrm{a}}$ & $\mathrm{LS}^{\mathrm{b}}$ & NO & \\
\hline${ }^{169} \mathrm{Lu}$ & $7 / 2^{+}$ & 2.297(13) & $2.2956(16)$ & & $1.001(6)$ \\
\hline${ }^{171} \mathrm{Lu}$ & $7 / 2^{+}$ & $2.305(12)$ & $2.2945(16)$ & $2.03(10)^{\mathrm{c}}$ & $1.005(5)$ \\
\hline${ }^{172} \mathrm{Lu}$ & $4^{-}$ & $2.893(15)$ & $2.8943(20)$ & $2.26(10)^{\mathrm{c}}$ & $1.000(5)$ \\
\hline${ }^{173} \mathrm{Lu}$ & $7 / 2^{+}$ & $2.280(12)$ & $2.2799(16)$ & $2.34(9)^{\mathrm{d}}$ & $1.000(5)$ \\
\hline${ }^{174 m} \mathrm{Lu}$ & $4^{-}$ & $1.497(10)^{\mathrm{e}}$ & $1.488(1)$ & $2.34(33)^{\mathrm{d}}$ & $1.006(7)$ \\
\hline${ }^{177 m} \mathrm{Lu}$ & $23 / 2^{-}$ & $2.337(13)$ & $2.3065(16)$ & $2.74(21)^{f}$ & $1.013(6)$ \\
\hline & & & & $3.3(3)^{\mathrm{g}}$ & \\
\hline & & & & $2.93(17)^{\mathrm{h}}$ & \\
\hline
\end{tabular}

${ }^{\mathrm{a}}$ This work.

${ }^{\mathrm{b}}$ Reference [10].

${ }^{\mathrm{c}}$ Reference [1].

${ }^{\mathrm{d}}$ Reference [2].

${ }^{\mathrm{e}}$ Reference [9].

${ }^{\mathrm{f}}$ Reference [3].

${ }^{\mathrm{g}}$ Reference [4].

${ }^{\mathrm{h}}$ Reference [14] (weighted average of Ref. [3] and [4]).

The result for the magnetic hyperfine splitting in $0^{\circ}$ geometry is

$$
\nu_{M}=79.2(2) \mathrm{MHz}
$$

Thus the quadrupole offset of the $0^{\circ}$-geometry resonance is

$$
(\bar{m}+1 / 2) \Delta \nu_{Q}=-9.32(24) \mathrm{MHz} .
$$

Taking into account the measurement temperature, model calculations yield

$$
\bar{m}=6.8(6) \mathrm{MHz} \text {. }
$$

Thus the quadrupole subresonance separation is found to be $\Delta \nu_{Q}=-1.27(12) \mathrm{MHz}$, yielding, as final result for the quadrupole interaction frequency,

$$
\nu_{Q}=-215(20) \mathrm{MHz} \text {. }
$$

For the nuclear moments we get

$$
\begin{gathered}
\mu\left({ }^{177 m} \mathrm{Lu}\right) / \mu\left({ }^{177} \mathrm{Lu}\right)=1.043(3), \\
Q\left({ }^{177 m} \mathrm{Lu}\right) / Q\left({ }^{177} \mathrm{Lu}\right)=+1.54(14) .
\end{gathered}
$$

and

$$
\begin{gathered}
\mu\left({ }^{177 m} \mathrm{Lu}\right)=2.337(13) \mu_{N}, \\
Q\left({ }^{177 m} \mathrm{Lu}\right)=+5.2(5) \quad \text { b. }
\end{gathered}
$$

\section{DISCUSSION}

Our results for the magnetic moments are listed in Table I, together with values from the literature.

The magnetic moments of the $I^{\pi}\left[N n_{z} \lambda\right]=7 / 2^{+}[404]$ ground states are almost constant between $A=169$ and 177 . Theoretical calculations of these magnetic moments by Ek- ström yielded $\mu=(2.20-2.21) \mu_{N}$ [15], in relatively good agreement with the experimental values. The theoretical calculations show also a very weak dependence of $\mu$ on the atomic number $A$, indicating that, between $A=169$ and 177, the wave function of the $7 / 2^{+}[404]$ proton is very weakly affected by additional neutron pairs.

The ground state configuration of ${ }^{172} \mathrm{Lu}$ is believed to be $\left\{\pi 7 / 2^{+}[404] \nu 1 / 2^{-}[521]\right\} 4^{-}$. For this configuration Ekström predicts $2.53 \mu_{N}$ [15], and Krane et al. predict $2.70 \mu_{N}[1]$, both values being slightly smaller than our experimental value, $\mu=2.893(15) \mu_{N}$. As the theoretical values depend strongly on the spin $g$ factor $g_{s}$, for which $g_{s}=0.6 g_{s}^{(\text {free })}$ has been taken, this difference should not be overinterpreted.

The configuration of the $23 / 2^{-}$state ${ }^{177 m} \mathrm{Lu}$ is believed to be $\left\{\pi 7 / 2^{+}[404] \nu 7 / 2^{-}[514] \nu 9 / 2^{+}[624]\right\} 23 / 2^{-}$. Taking experimental magnetic properties from neighboring nuclei with the respective single-particle configuration, the theoretical magnetic moment of a multiparticle state with $n$ particles can be calculated by

$$
\mu=\frac{I}{I+1}\left(\sum_{i}^{n} g_{K_{i}} K_{i}+g_{R}\right),
$$

where $g_{K_{i}}$ and $K_{i}$ are the $g$ factors and the $K$ quantum number for particle $i$, respectively. The collective $g$ factor $g_{R}$ in Eq. (28) may be taken as an average on the single-particle states, $g_{R}=(1 / n) \sum_{i}^{n} g_{R_{i}}$. Taking the magnetic properties of the neighboring nuclei ${ }^{177} \mathrm{Hf}\left(\nu 7 / 2^{-}[514]\right),{ }^{179} \mathrm{Hf}\left(\nu 9 / 2^{+}\right.$ [624]), and the ground state of ${ }^{177} \mathrm{Lu}\left(\pi 7 / 2^{+}[404]\right)$, the magnetic moment of ${ }^{177 m} \mathrm{Lu}$ is expected to be $\mu=2.40(6) \mu_{N}$ (see Ref. [4]), which is in good agreement with the experimental value. The existence of additivity-violating processes and/or different Coriolis mixing as had been proposed to explain the (wrong) magnetic moments from the NO measurements (Refs. $[3,4]$ ) can thus be excluded.

With the exception of ${ }^{173} \mathrm{Lu}$, the nuclear orientation results for the magnetic moments are in disagreement with the NMR-ON results of this work and the LS results of Ref. [10]. Thus it is once more confirmed that the interpretation of $\mathrm{NO}$ measurements is very problematic if the distribution of hyperfine interaction parameters for the impurity nuclei is unknwon. Here we would like to add that, in addition to the NMR-ON experiments described in this paper, we have performed NO measurements for all isotopes [16]. For these NO measurements the temperature was varied over a large scale - between $\sim 6$ and $60 \mathrm{mK}$ - by heating the mixing chamber of the dilution refrigerator. In all cases the effective magnetic hyperfine splitting $\nu_{M}^{(\mathrm{NO})}$ could be determined to an accuracy of $\sim 3 \%$. In most cases $\nu_{M}^{(\mathrm{NO})}$ was significantly smaller than the respective value from NMR-ON. The ratio $\nu_{M}^{(N O)} / \nu_{M}^{(\mathrm{NMR}-\mathrm{ON})}$ was found to be $0.79(2), 0.90(3), 0.82(2)$, $0.66(4), 0.87(2)$, and $1.05(4)$ for ${ }^{169} \mathrm{Lu},{ }^{171} \mathrm{Lu},{ }^{172} \mathrm{Lu}$, ${ }^{173} \mathrm{Lu},{ }^{177} \mathrm{Lu}$, and ${ }^{177 m} \mathrm{Lu}$, respectively [16]. Only if such a ratio were constant for all isotopes would the concept of using an effective (renormalized) magnetic hyperfine field be applicable. The large scattering of the experimental values for this ratio indicates that the distribution of $\mathrm{Lu}$ within the hcp Co host lattice depends extremely strong on experimental parameters, which are not under control. The NMR-ON 
TABLE II. Quadrupole moments of Lu isotopes of this work (NMR-ON), laser spectroscopy (LS) of Ref. [10], and nuclear orientation (NO).

\begin{tabular}{lcccccc}
\hline \hline Isotope & $I^{\pi}$ & \multicolumn{2}{c}{$Q[\mathrm{~b}]$} & & & $Q^{\text {(NMR-ON) }} / Q^{(\mathrm{LS})}$ \\
& & $\mathrm{NMR}^{\mathrm{N}}{ }^{\mathrm{a}}$ & $\mathrm{LS}^{\mathrm{b}}$ & $\mathrm{NO}$ & Theory $^{\mathrm{c}}$ & \\
\hline${ }^{169} \mathrm{Lu}$ & $7 / 2^{+}$ & $3.42(12)$ & $3.49(2)$ & & 3.38 & $0.98(3)$ \\
${ }^{171} \mathrm{Lu}$ & $7 / 2^{+}$ & $3.38(4)$ & $3.53(2)$ & & 3.45 & $0.96(2)$ \\
${ }^{172} \mathrm{Lu}$ & $4^{-}$ & $3.79(6)$ & $3.80(3)$ & & 3.81 & $1.00(2)$ \\
${ }^{173} \mathrm{Lu}$ & $7 / 2^{+}$ & $3.56(4)$ & $3.54(2)$ & & 3.53 & $1.01(1)$ \\
${ }^{177} \mathrm{Lu}$ & $7 / 2^{+}$ & $3.39(2)^{\mathrm{d}}$ & $3.39(3)$ & & 3.46 & \\
${ }^{177 m} \mathrm{Lu}$ & $23 / 2^{-}$ & $5.2(5)$ & $5.72(5)$ & $4.23(67)^{\mathrm{e}}$ & 5.77 & $0.91(9)$ \\
\hline \hline
\end{tabular}

This work

${ }^{\mathrm{b}}$ Reference [10].

${ }^{\mathrm{c}}$ Reference [15].

${ }^{\mathrm{d}}$ Reference [14], taken for calibration of the EFG.

${ }^{\mathrm{e}}$ Reference [17].

method, however, yields results which are to a large degree independent on the details of the distribution of the hyperfine interaction parameters. This can be seen from the ratio $\mu^{(\mathrm{NMR}-\mathrm{ON})} / \mu^{(\mathrm{LS})}$ which is given in column 6 of Table I. For the isotopes for which the quadrupole interaction could be resolved, the ratio is within the statistical error 1.0. For ${ }^{177 m} \mathrm{Lu}$, for which the parallel-perpendicular resonanceoffset method had to be applied because of the high spin of the isomer, the magnetic moment is by 1.3(6)\% larger than the LS value. The reason for this small discrepancy is probably due to the distribution of hyperfine fields in the vicinity of the hyperfine field on undisturbed substitutional lattice sites. In the resolved spectra this contribution appears as background and is thus less important. It should be pointed out, however, that despite this uncertainty due to the lacking knowledge of the hyperfine field distribution, the resulting uncertainty in the magnetic moment is only $\sim 1 \%$. This is remarkable, if one bears in mind that, for NO measurements, the unknown distribution of the hyperfine field introduces uncertainties larger by one to two orders of magnitude.

It is likely that the substitution behavior of $\mathrm{Lu}$ is similar for $\mathrm{Fe}$ and $\mathrm{ZrFe}_{2}$ as host lattices. Thus it is not astonishing that the NO results for the magnetic moments differ strongly from those obtained by NMR-ON and LS, even for the cases for which only ratios had been measured. The correct NO value for ${ }^{173} \mathrm{Lu}$ must thus be regarded as accidental. Probably, Lu is not the only case for which the NO technique fails. Thus, special attention should be given to the fact that there are elements for which the determination of the magnetic hyperfine splitting — to deduce either the nuclear magnetic moment or the magnetic hyperfine field - by the NO technique fails on principle.

Our results for the electric quadrupole moments are listed in Table II, together with values from the literature. Again, the agreement with the LS results is rather good. In addition, the theoretical quadrupole moments calculated by Ekström are in good agreement with the experimental quadrupole moments. Within the framework of the rotational model the spectroscopic quadrupole moment $Q$ for $K=I$ is connected with the intrinsic quadrupole moment $Q_{0}$ via

$$
Q=Q_{0} \frac{I(2 I-1)}{(I+1)(2 I+3)} .
$$

Within this model the ratio of quadrupole moments of the $23 / 2^{-}$isomeric state ${ }^{177 m} \mathrm{Lu}$ to the $7 / 2^{+}$ground state is assuming that both states can be described by the same intrinsic quadrupole moment $Q_{0}$ - expected to be

$$
Q\left(23 / 2^{-}\right) / Q\left(7 / 2^{+}\right)=1.668 .
$$

Our experimental value for this ratio is 1.54(14). Within the experimental uncertainty it is in good agreement with the theoretical value. Thus, according to our results, there is no obvious difference in the nuclear deformations of the ground state and the isomeric state. From NO measurement in a $\mathrm{Lu}$ single crystal, Oertel et al. reported $Q\left(23 / 2^{-}\right) / Q\left(7 / 2^{+}\right)=$ $1.25(20)$ [17], which indicated the deformation of the isomeric state to be smaller than that of the ground state. The LS ratio of these quadrupole moments is $Q\left({ }^{177 m} \mathrm{Lu}\right) /$ $Q\left({ }^{177} \mathrm{Lu}\right)=1.685(9) \quad[10]$, from which $Q_{0}\left(23 / 2^{-}\right)$ $/ Q_{0}\left(7 / 2^{+}\right)=1.010(5)$ can be deduced. This implies that the deformation of ${ }^{177} \mathrm{Lu}$ in the isomeric state is very similar to the deformation in the ground state.

Summarizing, the nuclear moments of the Lu isotopes determined with NMR-ON in hcp Co are in good agreement with the respective laser spectroscopy results. The nuclear moments can be well understood within the framework of Nilsson model calculations. There is no evidence for the existence of additivity-violating contributions in the nuclear magnetic properties of $\mathrm{Lu}$.

\section{ACKNOWLEDGMENTS}

We wish to thank H. Ratai for the preparation of the Co single crystals, E. Smolic, J. Hesol, and R. Schmid for experimental help, and G. Seewald for his help and dicussions in preparing the manuscript. Further we thank Dr. R. Neugart for the communication of the laser spectroscopy results prior to publication. This work was supported by the German Federal Minister for Research and Technology (BMFT) under Contract Nos. 06 TM 108 and by the DFG under Contract No. Ha 1282/3-3. 
[1] K. S. Krane, C. E. Olsen, and W. A. Steyert, Phys. Rev. C 13, 1295 (1976).

[2] K. S. Krane, C. E. Olsen, S. S. Rosenblum, and W. A. Steyert, Phys. Rev. C 12, 1999 (1975).

[3] K. S. Krane, C. E. Olsen, and W. A. Steyert, Phys. Rev. C 10, 825 (1974).

[4] E. Schoeters, R. E. Silverans, L. Vanneste, K. Freitag, and H. Hübel, Z. Phys. A 272, 203 (1975).

[5] J. Kern, A. Bruder, J. Cl. Dousse, M. Gasser, V.A. Ionescu, R. Lanners, B. Perny, B. Piller, Ch. Rheme, and B. Schaller, Phys. Lett. 146B, 183 (1984).

[6] A. Bruder, J. Cl. Dousse, S. Drissi, M. Gasser, V. A. Ionescu, J. Kern, B. Perny, M. Rast, and Ch. Rheme, Nucl. Phys. A467, 1 (1987).

[7] S. Drissi, J. Kern, and E. Hagn, Nucl. Phys. A512, 413 (1990).
[8] P. Herzog, U. Dämmrich, K. Freitag, C. D. Herrmann, and K. Schlösser, Hyperfine. Interact. 22, 167 (1985).

[9] B. Hinfurtner, E. Hagn, E. Zech, R. Eder, J. Kern, and the ISOLDE Collaboration, Phys. Lett. B 263, 29 (1991).

[10] U. Georg, W. Borchers, A. Klein, P. Lievens, R. Neugart, M. Neuroth, Pushpa M. Rao, Ch. Schulz, and the ISOLDE Collaboration, Z. Phys. A (to be published).

[11] R. Schmid, B. Hinfurtner, E. Hagn, E. Zech, and M. Deicher, Phys. Lett. A 174, 155 (1993).

[12] E. Hagn, Phys. Rev. B 25, 1521 (1982).

[13] H. Ratai, Ph.D. thesis, TU Munich, 1991.

[14] P. Raghavan, At. Data Nucl. Data Tables 42, 189 (1989).

[15] C. Ekström. Phys. Scr. 13, 217 (1976).

[16] C. König, Ph.D. thesis, TU Munich, 1991.

[17] D. Oertel, A. Kettschau, W. D. Brewer, and L. Vanneste, Z. Phys. A 310, 233 (1983). 